\title{
Network analysis of information flows to integrate in-vehicle information systems
}

\author{
John D. Lee* \\ Department of Mechanical and Industrial Engineering, \\ University of Iowa, Iowa City, IA 52242, USA \\ E-mail: jdlee@engineering.uiowa.edu \\ *Corresponding author
}

\section{Barry H. Kantowitz}

University of Michigan, UMTRI 2901 Baxter Road,

Ann Arbor, MI 48109, USA

E-mail: barrykan@umich.edu

\begin{abstract}
Many in-vehicle information systems (IVIS) are developed without consideration of how components need to be integrated. This can increase the cognitive load, errors and annoyance for drivers. This paper presents a taxonomy of IVIS functions and describes the information flows associated with these functions to show how a network analysis can guide integration. This analytic technique uses elements of graph theory to identify central functions and groups of functions that should be considered in system design. The analysis highlights potential dangers of failing to design integrated systems and the benefits of effective integration. Specifically, the analysis of information flows identifies highly central functions and groups of functions that are not obvious from the more traditional intuitive analysis of graphical representations.
\end{abstract}

Keywords: network analysis; function analysis; system integration; driver distraction; IVIS; cognitive task analysis; information architecture.

Reference to to this paper should be made as follows: Lee, J.D. and Kantowitz, B.H. (2005) 'Network analysis of information flows to integrate in-vehicle information systems', Int. J. Vehicle Information and Communication Systems, Vol. 1, Nos. 1/2, pp.24-43.

Biographical notes: John D. Lee is an Associate Professor of Industrial Engineering at the University of Iowa. He has a background in engineering and psychology, with a $\mathrm{PhD}$ in mechanical engineering from the University of Illinois at Urbana-Champaign. His research interests include interface design, human adaptation to technological innovation and human performance modelling.

Barry Kantowitz is a Professor of Industrial and Operations Engineering, Professor of Psychology, and Director of the Transportation Research Institute at the University of Michigan. When not doing all of the preceding, he occasionally gets an opportunity to do some research. 


\section{Introduction}

Recent technological advances have made a wide variety of information systems possible for cars and trucks. New sensor capabilities, global positioning systems, the internet and wireless communication constitute four particularly important technologies capable of dramatically altering the driving task (Jameel et al., 1998). In-vehicle information systems (IVIS) can provide drivers with an array of information; MP3 music catalogues, e-mail, route guidance, and hazard warnings represent a few of the many emerging mobile computing applications for automotive use. This information technology when properly deployed, can increase productivity, safety, and comfort. Improperly deployed technology can degrade safety and annoy drivers.

New systems are developed frequently without careful consideration of how they integrate with other systems. For example, navigation aids are installed on ships (Lee and Sanquist, 2000), functions are added to flight management systems (Sarter and Woods, 1995) and displays of medical device displays are combined (Cook, Woods and Howie, 1990) without careful attention to how the information from these systems should be integrated. Failure to properly integrate these systems has led to substantial confusion and frustration. As demonstrated by the recent controversy and confusion regarding the 700 features of the BMW iDrive, failure to integrate IVIS functions can cause problems (Norman, 2003).

The general concept of functional integration has significant potential for enhancing human-system performance. However, few analytic tools have been developed to support functional integration. Functional integration involves analysis of the information required by each function, the information produced by each function and how the information flows need to be supported and coordinated. The information inputs and outputs of the various system functions define links between functions that can either be identified and supported by designers or discovered and accommodated by drivers. Drivers who are forced to 'finish the design' by providing necessary links between functions, may experience increased cognitive load, annoyance, and dissatisfaction with the system (Rouse and Cody, 1988). One factor contributing to poorly integrated design is the large number of companies contributing to the devices that go into cars. Each device is designed independent of the others and there is no central entity that takes responsibility for the combined effects of each function. Another factor that contributes to the lack of integration is the relative lack of analytic tools to support designers in creating integrated systems, many systems are poorly integrated and subject drivers to the error-prone and laborious task of finishing the design. For example, typical route guidance systems are disconnected from the address book and appointments in a driver's PDA so that the driver must finish the design and enter the addresses manually. This paper demonstrates computational tools that can analyse the information flows between functions, and shows how these analyses might be used to improve IVIS.

Computational approaches to examining information flows are critical because the graphics that frequently document information flows are often incomprehensible and at best provide only a qualitative description of the system (Kirwan, 1992). Typically, each function is represented by an ellipse labelled with the function name and information flows are usually designated using arrows. These graphics provide a visual representation that can sometimes promote insights; however, as they become more complex, they grow harder to draw and comprehend. Alternately, an information flow may be represented as 
a matrix in which information flows between functions are summarised as functions arrayed against functions. In each instance where information flows from one function to another, the cell in the matrix contains a ' 1 ', otherwise it contains a ' 0 '. Each function can have inputs and outputs. The inputs for the function are identified by the contents of the column associated with the function. The outputs are identified by the contents of the row associated with the function. Information flows from the functions in the rows to the functions in the columns. A benefit of the matrix approach is that it easily scales to accommodate increasingly complex systems, in contrast to graphical representations, which can quickly become unmanageable. A matrix representation also enables a range of mathematical analyses capable of revealing relationships that may not be obvious in graphic representation. Figure 1 shows both graphical and matrix representations of a hypothetical set of functions. More formally, Figure 1 represents a digraph, or directional graph, in which each function is a vertex and each arrow represents an edge. The matrix of vertices and corresponding edges can be represented as a matrix $\mathrm{X}$, where the element $x_{i j}=1$ when an edge connects two vertices and $x_{i j}=0$ when vertices are unconnected. Treating functions and information flows as a digraph makes the application of well-established graph theory techniques developed to study social networks possible (Borgatti, Everett and Freeman, 1992; Luce and Perry, 1949; Wasserman and Faust, 1994) and engineering design (Kusiak, 1999). This diagram shows that even for a system of only five functions, the graphical representation can become confusing and that the formalisms of graph theory can provide a useful tool to address this complexity.

Figure 1 Graphical and matrix representations of functions and information flows

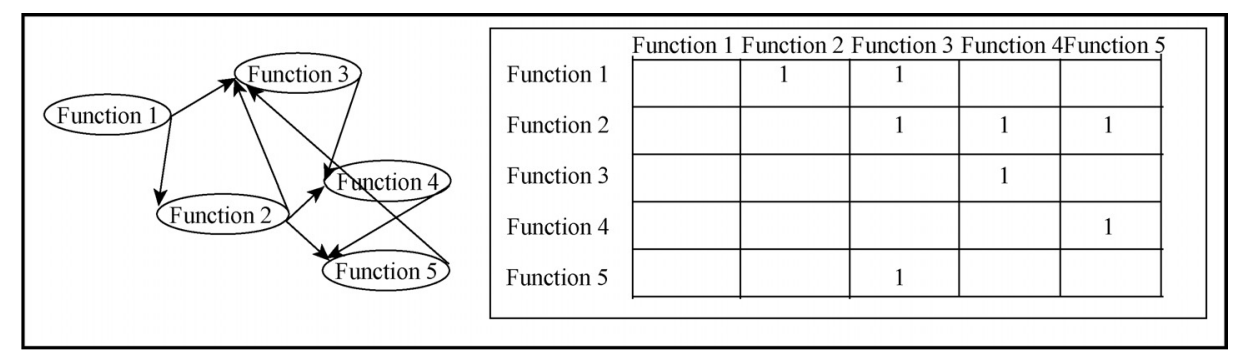

The objective of this paper is to describe how network analysis might be used to analyse IVIS functions to support driver-centred integration. Figure 2 provides an overview of the function analysis and integration process. It begins by defining the functions and their information flows. The information flows among the functions are then represented in a matrix form. A series of matrix operations can then be performed to assess the centrality of each function and to identify groups of related functions. Identification of highly central functions and groups of related functions then provides a technical basis to guide design decisions.

\section{IVIS functions and information flows}

The first step in considering functional integration is to develop a functional description of the system. Such a description represents the generic capabilities, requirements, and processes of a given system (Rasmussen, 1985). It does not, however, specify the physical mechanisms or physical configuration. A functional description, therefore, 
explains how functions can be used to satisfy system requirements without identifying the specific, physical mechanisms used to implement the design. One way to clarify the nature of a functional description is to place it in the context of other ways of describing the same system. Systems can be described at various levels of abstraction and aggregation (Rasmussen, 1985, 1986; Vicente, 1999). The dimension of aggregation defines the level of detail or scope included in the system description. The dimension of abstraction specifies the relationship between the purpose of the system and its physical implementation. Function integration depends on a description of functions and information flows, whereas physical integration depends on a description of the physical displays and controls with which the drivers interact.

Figure 2 Analysis and interpretation of information flows

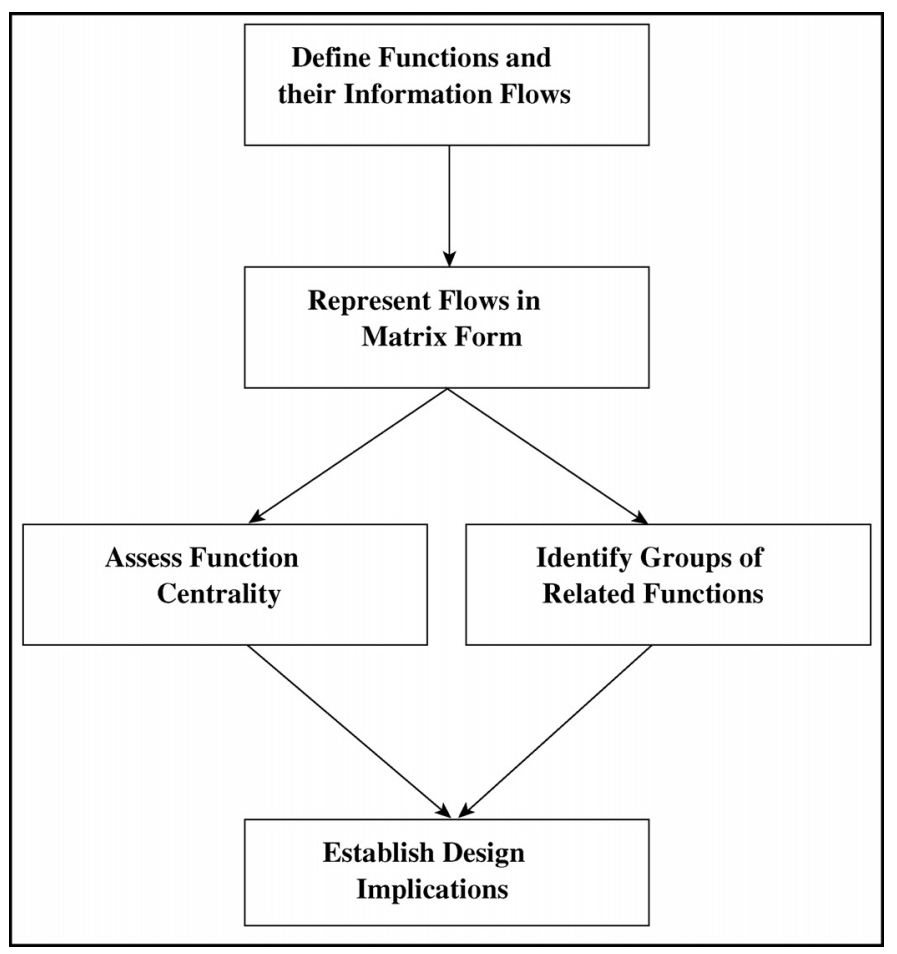

A functional description is more concrete than a description of system purposes and high-level performance objectives, but less concrete than a description of the physical layout of the system. The functional description identifies how system objectives are achieved and indicates why physical features of the system need to be present. Most systems serve several purposes and include many functions, which can be implemented in many different ways. With IVIS, trip planning and route guidance enhance driver mobility, whereas interactive entertainment and real-time communication focus on driver comfort and productivity. These functions can be realised in many different physical forms, such as icons on LCD panels, a computer-generated voice or the sensor systems that provide the data for these displays. Lee (1997) provides a detailed description of IVIS at several levels of abstraction and aggregation. Table 1 summarises IVIS functions and shows example outputs of each function. 
Table 1 A summary of IVIS functions

\begin{tabular}{|c|c|c|}
\hline IVIS Capabilities & Functions & Example Output \\
\hline \multirow[t]{9}{*}{ Routing and navigation } & 1.1 Trip planning & Length of route \\
\hline & $\begin{array}{l}\text { 1.2 Multi-mode travel coordination } \\
\text { and planning }\end{array}$ & Arrival time at destination \\
\hline & $\begin{array}{l}\text { 1.3 Pre-drive route and destination } \\
\text { selection }\end{array}$ & Route to destination \\
\hline & 1.4 Dynamic route selection & New route \\
\hline & 1.5 Route guidance & Street name to turn on \\
\hline & 1.6 Route navigation & Location on route \\
\hline & 1.7 Automated toll collection & Toll cost \\
\hline & 1.8 Route scheduling & Order of destinations \\
\hline & 1.9 Post-trip summary & Trip duration \\
\hline \multirow[t]{4}{*}{ Motorist services } & 2.1 Broadcast services/attractions & Lodging location \\
\hline & 2.2 Services/attractions directory & $\begin{array}{l}\text { Directory (index of yellow } \\
\text { pages) }\end{array}$ \\
\hline & 2.3 Destination coordination & $\begin{array}{l}\text { Location of and distance to } \\
\text { restaurant }\end{array}$ \\
\hline & 2.4 Delivery related information & $\begin{array}{l}\text { Scheduled pickup and } \\
\text { delivery times }\end{array}$ \\
\hline \multirow[t]{3}{*}{ Augmented signage } & 3.1 Guidance sign information & Route markers \\
\hline & 3.2 Notification sign information & Sharp curve ahead \\
\hline & 3.3 Regulatory sign information & Speed limit \\
\hline \multirow[t]{7}{*}{ Safety and warning } & 4.1 Immediate hazard warning & $\begin{array}{l}\text { Emergency vehicle stopped } \\
\text { ahead }\end{array}$ \\
\hline & 4.2 Road condition information & Traffic congestion ahead \\
\hline & 4.3 Automatic aid request & $\begin{array}{l}\text { Inform driver aid has been } \\
\text { requested }\end{array}$ \\
\hline & 4.4 Manual aid request & Display status of aid request \\
\hline & 4.5 Vehicle condition monitoring & Low oil pressure alert \\
\hline & 4.6 Driver monitoring devices & Estimate of driver alertness \\
\hline & 4.7 Sensory augmentation & Image of road ahead \\
\hline \multirow{7}{*}{$\begin{array}{l}\text { Collision avoidance and vehicle } \\
\text { control }\end{array}$} & 5.1 Forward collision avoidance & Imminent collision warning \\
\hline & $\begin{array}{l}\text { 5.2 Road departure collision } \\
\text { avoidance }\end{array}$ & $\begin{array}{l}\text { Virtual rumble strips } \\
\text { through seat vibration }\end{array}$ \\
\hline & $\begin{array}{l}\text { 5.3 Lane change and merge collision } \\
\text { avoidance }\end{array}$ & $\begin{array}{l}\text { Graded alert based on lane } \\
\text { position }\end{array}$ \\
\hline & 5.4 Intersection collision avoidance & Vehicle incursion \\
\hline & $\begin{array}{l}5.5 \text { Railroad crossing collision } \\
\text { avoidance }\end{array}$ & Presence of train \\
\hline & 5.6 Backing aid & Degree of brake engagement \\
\hline & 5.7 Advanced cruise control & Desired speed setting \\
\hline $\begin{array}{l}\text { Driver comfort, communication, } \\
\text { and convenience }\end{array}$ & 6.1 Real-time communication & Incoming cellular phone call \\
\hline
\end{tabular}


Table 1 A summary of IVIS functions (Continued)

\begin{tabular}{lll}
\hline IVIS Capabilities & \multicolumn{1}{c}{ Functions } & \multicolumn{1}{c}{ Example Output } \\
\hline 6.2 Asynchronous communication & List of email messages \\
6.3 Contact search and history & Name of last person called \\
6.4 Entertainment and general & Track number of current CD \\
information & \\
6.5 Interactive entertainment & Points accrued in game \\
6.6 Heating, ventilation, & Heater setting \\
& air-conditioning, and noise & \\
6.7 Automatic system configuration & Seat and mirror setting \\
\hline
\end{tabular}

Table 2 shows a matrix of likely information flows between IVIS functions based on current evolution of IVIS functions. The information flows define links between functions that enable efficient operation of the overall system. For example, information that specifies the address of restaurants flows from services and attractions directory to the pre-drive route and destination selection.

Table 2 A information flows between IVIS functions

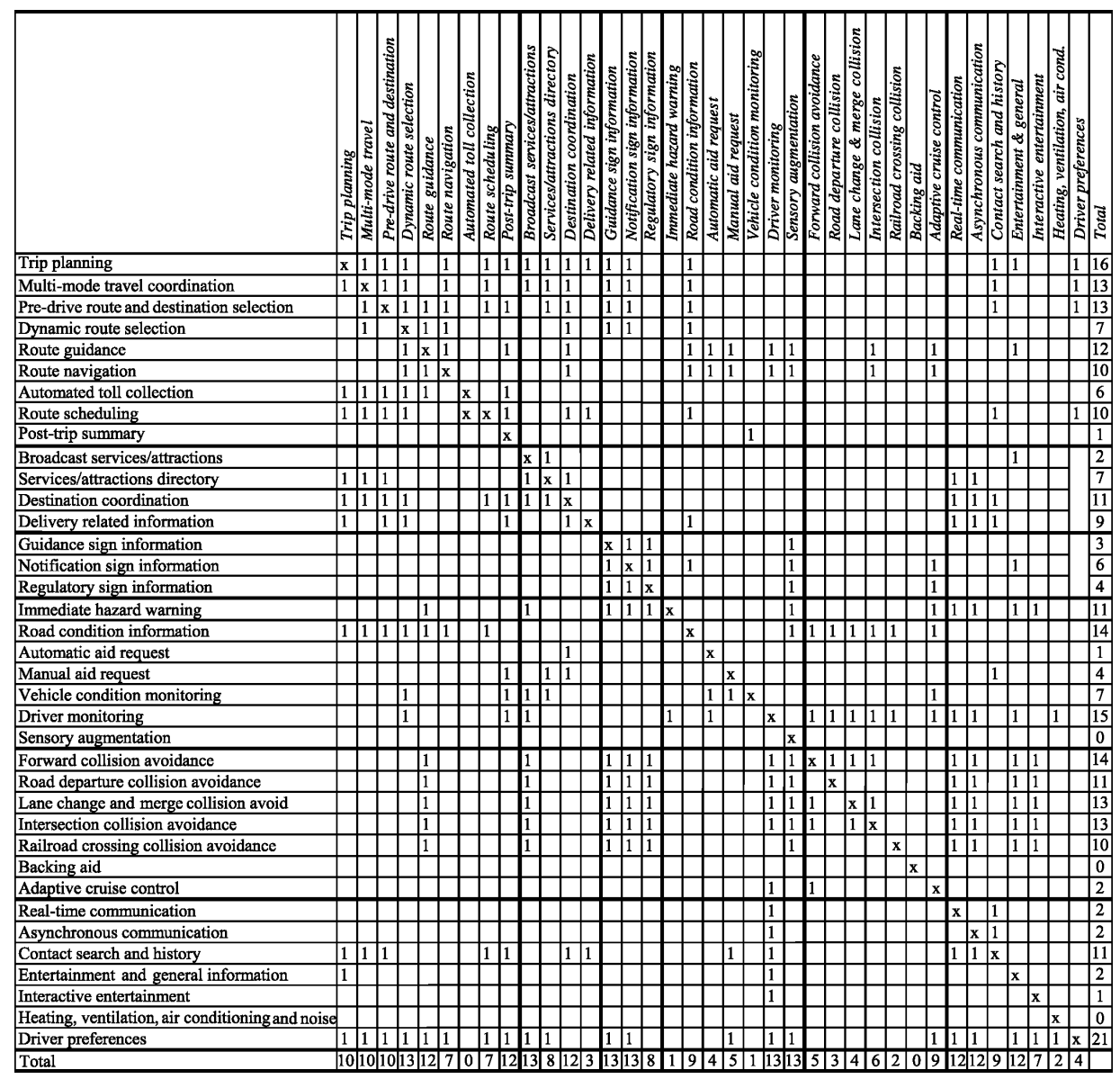


The entries in Table 2 summarise several different types of information flows, including information that would otherwise need to be entered by the driver, such as destination input for route navigation. The information flows also include information from a function that provides the context for interpreting the output of another function. For example, driver-monitoring data provides a context for interpreting emerging collision situations. Another type of information flow prioritises messages from the various systems. For instance, if information is shared between forward object collision warning and a real-time communication (e.g., a cell phone), then incoming calls could be deferred in the event of an impending collision.

Generating the data represented in Tables 1 and 2 requires substantial knowledge of the domain and a detailed analysis of each function. The entire analysis relies on the accuracy of the data in these two tables. The list of functions in Table 1 was developed by the lead author and then reviewed by several domain experts. The list of functions and their definitions were refined to address the comments. The information flows for each function were defined and verified in an iterative manner by the two authors. In this way, the process of defining functions, refining these definitions and then clarifying information flows often requires an approach similar to the Delphi method. The Delphi method can effectively integrate the perspectives of several experts (Linstone and Turoff, 1975; Turban and Tan, 1993). This is particularly important when the system of interest is at the early stages of conceptual design. With better defined systems, the engineering specifications of each function can provide the data shown in Table 2.

Even with a moderately complex system such as this, it is not obvious how these functions should be integrated, thus meriting a systematic analysis. The matrix of information flows between functions defines a network of interconnected elements. Many techniques have been developed to aid understanding of the relationships between network elements and these may also apply to the task of integrating IVIS functions. One particularly useful class of network analysis techniques has emerged from the study of social groups, in which the network represents relationships between people (Bavelas, 1948; Freeman, 1978). Two types of network analysis techniques are particularly relevant. One type examines the centrality of each function, i.e., the degree to which a function is linked to other functions. Another type of analysis identifies groups of related functions. Highly central functions and cohesive groups of functions are particularly critical for the development of an integrated IVIS. Both of these analyses can provide useful insight into the integration of IVIS. UCINET IV is a software tool for network analysis that uses a matrix of information flows as input and produces summary data that describe how functions are related. UCINET IV was used for the majority of the network analyses presented in this article (Borgatti, Everett and Freeman, 1992).

\subsection{Input and output centrality}

Function centrality can be expressed in terms of both input and output centrality. Input centrality refers to the degree to which a function receives input from other functions. Functions with high input centrality depend on other functions to be useful. Output centrality refers to the degree to which functions provide input to other functions. Functions with high output centrality facilitate and support many other functions. In Figure 2, Function 2, which provides inputs to three functions, has a high output centrality, whereas Function 3 has a high input centrality, since it requires input from 
three other functions. The matrix representation of large systems helps identify highly central functions that might otherwise be obscured by complex graphical representation.

Nieminen (1974) defined centrality as a function of the degree of a vertex. For any matrix $X$, the degree of any function, represented as a vertex, $x_{i j}$, is the sum of the edges that connect the vertex to other vertices. $X_{(\mathrm{i}, \mathrm{j}}$ ) $=1$ when the two vertices (functions) are linked by an edge (information flow). Equation (1) represents the calculations associated with Nieminen's metric of input centrality and Equation 2 represents output centrality calculations:

$$
\begin{aligned}
& \text { Input_Centrality }_{j}=\sum_{i=1}^{n} X(i, j) \\
& \text { Output_Centrality }_{j}=\sum_{j=1}^{n} X(i, j)
\end{aligned}
$$

This measure of centrality is simply the number of functions using an input from or providing an output to that function, and is a simple estimate of centrality that is dependent on network size. Input centrality of each function in Figure 2 is simply the sum of each column of the table and output centrality is simply the sum of each row. The input and output centrality of each function are shown at the bottom and right side of Figure 3. Dividing by the number of functions in the network normalises these values. Although the normalised value enables comparisons across networks of different sizes, a simple count of information flows to/from each function as an estimate of input and output centrality ignores the relative importance of the source of the information flow. For example, consider two functions, one of which receives input from seven functions and the other from five functions. A simple normalised count identifies the function with seven inputs as having a higher input centrality. This calculation would be misleading if each of the five input functions in the second case receives input from six other functions and each of the seven input functions in the first case receives input from only two other functions.

Figure 3 Graphical and matrix representations of functions with high input (Function 3) and output centrality (Function 2)

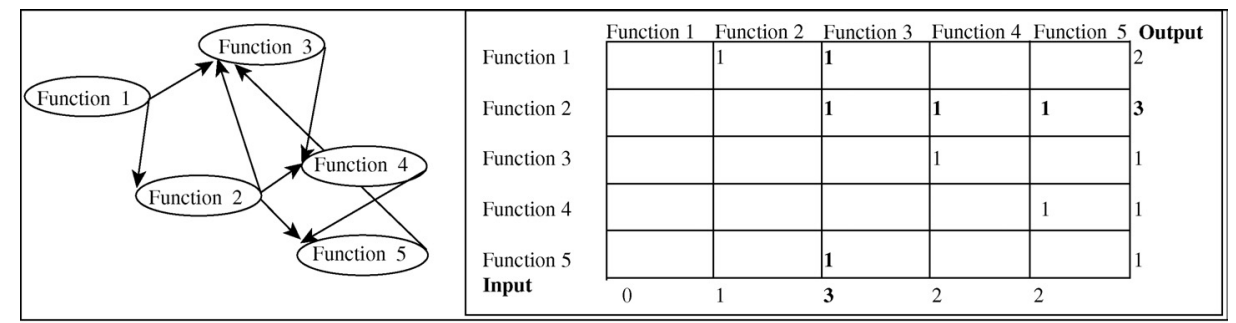

The results shown in Tables 3-6 have been generated using a technique that considers the relative contribution of the various functions by differentially weighting inputs and outputs according to overall network interconnections. This approach is based on an interesting property of matrix operations for which the elements of $X^{r}$ represent the number of paths of length $r$ between vertices in the graph. $X^{r}$ represents the matrix $X$ raised to the power of $r$. When $r=1$, the matrix represents information flows from 
directly connected functions. When $r=2$, the matrix represents information flows from functions that must first pass through one intermediate function.

Information flows to that pass through one or more intermediary functions are unlikely to be as influential as those that flow directly to that function. The degree of influence of intermediary information flows on a function should, however, be determined and used in the calculation of centrality. This can be accomplished using an attenuation factor that reduces the influence of information flows that require longer paths to reach a given function. Equation 3 calculates the total information flow to a function, weighted by the proximity of the connection. If an information flow passes through three other functions before reaching a particular function then it is weighted less heavily than one that passes through just one intermediary function. The attenuation factor, $m$, weights the indirect information flows in the calculation of the matrix of total information flows between functions $\left(X_{t}\right)$. As shown in Equation 3, the attenuation factor weights the information flows such that with an attenuation factor of 0.10 the direct flows are counted as 0.1 and those that are separated by a single intermediary function are weighted as 0.01 . Once the total information flow matrix was calculated using Equation 3, then Equations 4 and 5 were used to calculate the input and output centrality. The attenuation factor should be smaller than the reciprocal of the absolute magnitude of the maximum eigenvalue (Borgatti, Everett and Freeman, 1992). This analysis used a value that was $20 \%$ smaller than that bound; $m$ was set to 0.10 .

$$
\begin{aligned}
& X t=\sum_{r=1}^{n-1} \mu^{r} X^{r} \\
& \text { Input_Centrality }_{j}=\sum_{i=1}^{n} X t(i, j) \\
& \text { Output_Centrality }_{i}=\sum_{j=1}^{n} X t(i, j)
\end{aligned}
$$

Figure 4 summarises the input and output centrality of the 37 functions listed in Table 2. Input and output centrality combine to define the total centrality of a function, as represented by the total length of the bars.

Table 3 lists the functions with the ten highest levels of input centrality. Functions with high levels of input centrality demonstrate a higher degree of dependence on information from other functions. For example, driver monitoring, destination coordination, and dynamic route selection operate effectively only when provided with information from several other functions. In particular, driver monitoring requires knowledge of the driver's use of other in-vehicle functions to estimate the driver's cognitive state. Input centrality is particularly important because it identifies functions that might make substantial demands on the driver to enter information if these information flows are not automated. Dynamic route selection operates as the driver is driving towards the destination so data entry could be hazardous.

Table 4 lists the functions with the ten highest levels of output centrality. The high output centrality of these functions indicates their importance in supporting the proper performance of other functions. For example, trip planning could produce substantial information that could be used by other functions, such as dynamic route selection, in 
generating turn-by-turn instructions. Likewise, driver preferences tailors the information flow to the particular desires of individual drivers and so could affect the operation of many functions.

Figure 4 The input and output centrality of the in-vehicle information system functions

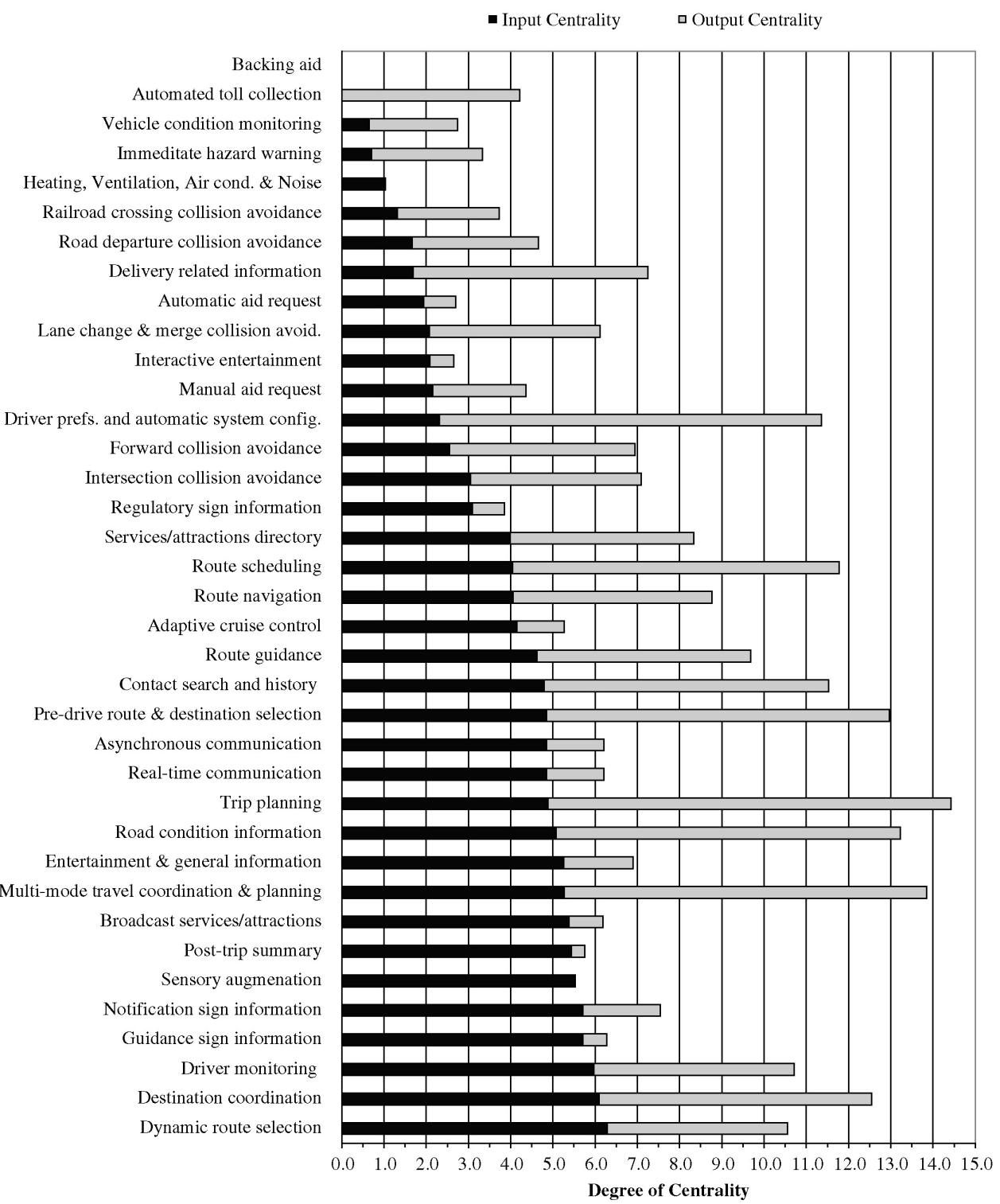

Tables 5 and 6 combine input and output centrality measures to reveal important relationships between the functions not discernible from either analysis on its own. In Table 5, individual input and output centralities are summed to identify total centrality. Functions with a high total centrality, such as trip planning, road condition information and driver monitoring, are identified as critical elements of IVIS that deserve particular 
attention in system design. In Table 6, input and output centralities are subtracted to identify the 'leverage' of individual functions. The 'leverage' metric identifies functions that have a high output centrality relative to input centrality. These functions require input from relatively few functions, but provide it to many. In this regard, the driver preferences function is substantially more important than any other, which is consistent with the fact that this function receives information from the driver and distributes it to a wide range of other functions. This is a particularly useful metric, because it identifies those functions that have few information sources, but are likely to support many other functions. For these functions it is particularly important to ensure that their information sources are well-supported otherwise many functions could suffer.

Table 3 The ten functions with the highest levels of input centrality

\begin{tabular}{lc}
\hline Function & Input Centrality \\
\hline Dynamic route selection & 6.29 \\
Destination coordination & 6.09 \\
Driver monitoring & 5.97 \\
Guidance sign information & 5.71 \\
Notification sign information & 5.71 \\
Sensory augmentation & 5.53 \\
Post-trip summary & 5.44 \\
Broadcast services/attractions & 5.38 \\
Multi-mode travel coordination & 5.27 \\
Entertainment and general information & 5.26 \\
\hline
\end{tabular}

Table 4 The ten functions with the highest levels of output centrality

\begin{tabular}{lc}
\hline Function & Output Centrality \\
\hline Trip planning & 9.54 \\
Driver preferences & 9.04 \\
Multi-mode travel coordination and planning & 8.58 \\
Road condition information & 8.15 \\
Pre-drive route and destination selection & 8.13 \\
Route scheduling & 7.72 \\
Contact search and history & 6.74 \\
Destination coordination information & 6.45 \\
Delivery related information & 5.56 \\
Route guidance & 5.07 \\
\hline
\end{tabular}

\subsection{Cliques and clusters}

In addition to centrality estimates of individual functions, identifying how information flows can define groups of functions is also an important design consideration. Grouping functions may help identify synergistic combinations of functions that, if integrated, could greatly enhance IVIS capabilities. These groups represent meta-level functionality 
that can be used to guide system definition. For this reason, interconnected groups of functions can provide an organising framework for in-vehicle system functionality and the driver interface. Two types of network analyses were performed to examine how information flows between functions. The first analysis identifies cliques, while the other identifies clusters. Each approach employs different criteria to group functions - together they provide convergent information regarding groups of functions.

Table 5 The ten functions with the highest level of total centrality

\begin{tabular}{lc}
\hline Function & Combined Centrality \\
\hline Trip planning & 14.4 \\
Multi-mode travel coordination and planning & 13.8 \\
Road condition information & 13.2 \\
Pre-drive route and destination selection & 13.0 \\
Destination coordination & 12.5 \\
Route scheduling & 11.8 \\
Contact search and history & 11.5 \\
Driver preferences & 11.4 \\
Driver monitoring & 10.7 \\
Dynamic route selection & 10.6 \\
\hline
\end{tabular}

Table 6 The ten functions with the highest level of 'everage' (output centrality minus input centrality)

\begin{tabular}{lc}
\hline Function & Leverage Centrality \\
\hline Driver preferences and automatic system configuration & 6.72 \\
Trip planning & 4.66 \\
Automated toll collection & 4.22 \\
Delivery related information & 3.88 \\
Route scheduling & 3.67 \\
Multi-mode travel coordination & 3.32 \\
Pre-drive route and destination selection & 3.28 \\
Road condition information & 3.08 \\
Lane change and merge collision avoidance & 1.96 \\
Contact search and history & 1.94 \\
\hline
\end{tabular}

A clique is a formal description of the density of links between nodes in a network. Defined as a coherent grouping of functions connected by information flows, a clique represents a maximally complete sub-graph (Luce and Perry, 1949). In other words, a clique is a grouping of vertices that are directly linked to each other by an edge. Each member of a clique must have at least as many connections to other functions as there are members in the clique. For example, in a clique of three nodes, each node must be linked to all other nodes in the clique, in addition to any other links to functions in the network that are not members of the clique. Thus, a clique is a group of functions that share information directly with each other. This information sharing does not need to be 
bi-directional - a one-way sharing of information is sufficient to form a clique. Figure 3 shows how Functions 1, 2 and 3 of the hypothetical system satisfy the requirements of a clique.

The functions in Table 2 were analysed to identify cliques of at least six members. The clique size greatly affects the number of cliques that can be identified. As the number of clique members increases the number of groups of functions that meet the definition of a clique decline dramatically. The particular form of the relationship between clique size and the number of cliques depends on the characteristics of the system. The number of cliques in system that has broad interconnections will decline more slowly as clique size increases compared to a system composed of localised connections. This network of 37 functions contains 19 six-member cliques, as summarised in Table 7. The large number of relatively large cliques demonstrates that IVIS functions are highly interconnected and merit careful analysis to ensure functional integration.

Table $7 \quad$ A summary of the cliques with more than six members

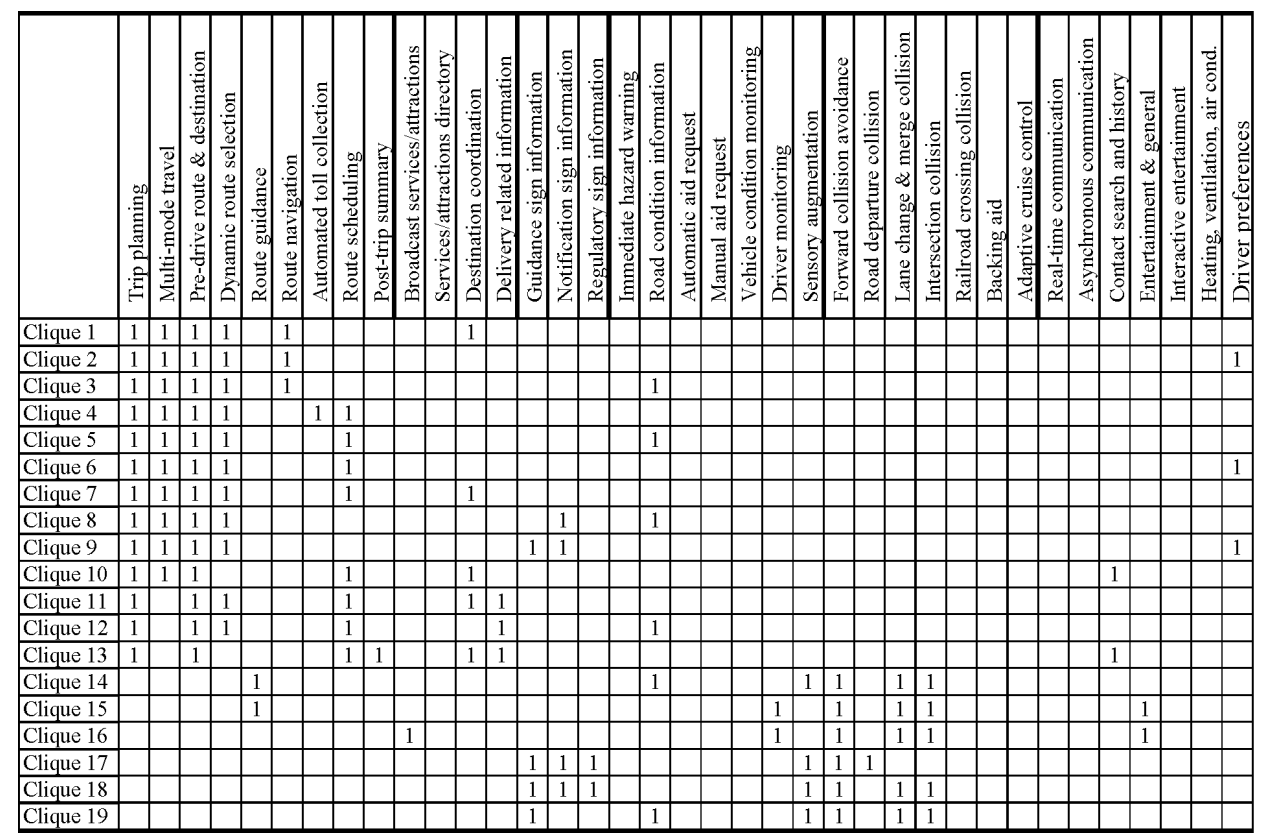

An inspection of clique membership pattern reveals two distinct groups. The first group, listed at the top of Table 7, focuses on planning and route choice functions. The second group, listed at the bottom of Table 7 (cliques 14-19), focuses on moment-to-moment driving functions, such as route guidance, collision warning, and entertainment. Interestingly, only two functions are included in both groups: notification sign and road condition. As expected, each of these functions plays an important role in both trip planning and vehicle control. These results are important from a design perspective because they identify groups of functions that must be directly linked by information flows and are likely to be used together by the driver. In addition, the two groups of cliques identify a fundamental distinction between functions that should be considered in design. The planning functions may need to link with information sources and 
planning functions outside the vehicle, whereas the other group of functions must be linked to vehicle control.

Cluster analysis identifies functions linked by information flows that are not completely interconnected as required for membership in a clique. Although it does not impose requirements as strict as clique membership, cluster analysis extracts groups of functions that tend to share information. A cluster analysis maximises a cost function based on the extent to which a group of functions consists of clique-like structures. Thus, cluster analysis identifies groups of functions in a manner somewhat analogous to factor analysis. Specifically, UCINET IV uses a tabu search method to maximise a cost function (Borgatti, Everett and Freeman, 1992). A tabu search method guides an optimisation algorithm to avoid local optima by forbidding moves to points in the solution space that have been visited previously (Glover, 1977). Table 8 summarises five clusters of IVIS functions listed in Table 2. The clusters share some similarities with the grouping based on general IVIS capabilities. This is not surprising because the original organisation was based on general functional similarity. Importantly, many of the functions cluster into groups different than the IVIS capabilities. These differences have important design implications. For example, driver preferences was found to belong to a cluster that includes dynamic route selection and route navigation. Traditionally, the driver preferences function has been more commonly associated with vehicle climate control, seat adjustment, and radio settings, but this analysis shows that it may play an important role in many other functions including navigation-related functions. Because the cluster analysis relaxes the strict requirement of complete interconnection between functions, it generates complementary results to that based on cliques. The cliques identify functions that require information flows to link them, whereas the cluster analysis relaxes this requirement and identifies groups of functions that are relatively tightly interconnected by information flows.

\section{Discussion}

Analyses of function centrality and function clusters demonstrate the potential benefits of network analysis. These analyses can make important contributions to the design of IVIS by identifying how functions should be developed, linked and integrated. In even moderately complex systems traditional approaches that rely upon graphical approaches and intuition will likely fail to produce these insights.

\section{$3.1 \quad$ Functional integration in design}

Although traditional engineering design acknowledges the need for hardware and software integration, such integration does not always consider how to integrate information flows to support the driver. For example, integration efforts often focus on the physical integration of information systems in a common input device or display. While this level of integration might provide benefits to the driver by eliminating redundant components and an array of potentially confusing devices, it might not enhance driver performance. A complex array of functions combined in a single physical location can actually hinder driver performance by making the formerly directly accessible functions available only through menu selections and multifunction buttons. 
For example, the volume control of a conventional car radio is a single function knob that is easily accessible to the driver. If the radio is physically integrated with a navigation aid and cellular telephone then the physical controls will be shared by the three systems. In such a physically integrated configuration, changing the radio volume may only be accomplished by first selecting the radio function, a process that could increase errors and glances away from the road. Hence, physical integration of a complex set of separate devices using a simple interface can mask a complex array of options (Woods, 1994). The apparent simplicity of physical integration may actually increase the complexity that confronts the driver.

Table 8 A summary of information flows and the five clusters

\begin{tabular}{|c|c|c|c|c|c|c|c|c|c|c|c|c|c|c|c|c|c|c|}
\hline 1 Trip planning & 1 & & $1 \mid 1$ & 1 & 1 & 11 & 1 1 1 & & & & & & 1 & & & & & \\
\hline 1 Multi-mode travel coordination & 1 & & 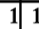 & $1]$ & 1 & 1 & $\begin{array}{lll}1 & 1 & \\
1\end{array}$ & & & & & & 1 & & & & & 1 \\
\hline 1 Pre-drive route $\&$ destination selection & & & \begin{tabular}{l|l}
1 & 1 \\
\end{tabular} & 1 & 1 & 1 & 1 & & & & & & & & & & & \\
\hline 1 Dynamic route selection & & 1 & $\left.\begin{array}{|l|lll}1 & 1 & 1\end{array}\right)$ & 1 & & & 1 & & & & & & & & & & & \\
\hline 1 Road condition information & 1 & 11 & \begin{tabular}{l|l}
1 & 1 \\
\end{tabular} & 1 & 1 & & & 1 & & $1 \mid 1$ & 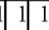 & 1 & & & 1 & & & \\
\hline 1 Route navigation & & & \begin{tabular}{l|l}
1 & 1 \\
\end{tabular} & 1 & & & 1 & & & & 1 & 1 & & & & & & \\
\hline 1 Services/attractions directory & 1 & 1 & & T1 & & & 1 & & & & & & & & 1 & & & \\
\hline 1 Route scheduling & 1 & 11 & \begin{tabular}{l|l}
1 & 1 \\
\end{tabular} & & 1 & & 1 & & & & & & & 1 & & & & \\
\hline 1 Delivery related information & 1 & 1 & \begin{tabular}{l|l}
1 & 1 \\
\end{tabular} & & & 1 & 1 & & & & & & & & & & & \\
\hline 1 Driver preferences & 1 & 1 & 1 & $1]$ & 1 & 1 & & & & & $\overline{7}$ & \begin{tabular}{l|l}
1 & 1 \\
\end{tabular} & 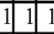 & & 1 & & & \\
\hline 1 Contact search and history & 1 & 11 & & & 1 & & 1 & & & & & & & & & & & \\
\hline 1 Destination coordination & 1 & & 1 & & 1 & & 1 & & & & & & & & 1 & & & \\
\hline 2 Route guidance & & & & & & & & 1 & & & 1 & 1 & $1|1|$ & & & & & \\
\hline 2 Guidance sign information & & & & & & & & 1 & 1 & & 1 & & & & & & & \\
\hline 2 Road departure collision avoidance & & & & & & & & 1 & 1 & & 1 & & 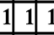 & & 1 & & & \\
\hline 2 Regulatory sign information & & & & & & & & 1 & 1 & & 1 & 1 & & & & & & \\
\hline 2 Lane change $\&$ merge collision avoid. & & & & & & & & $1 \mid 1$ & & \begin{tabular}{|l|l|l|l|l}
1 & 1 \\
\end{tabular} & $1 \quad 1$ & & 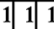 & & 1 & & 1 & \\
\hline 2 Intersection collision avoidance & & & & & & & & $\begin{array}{lll}1 & 1 \\
\end{array}$ & 1 & \begin{tabular}{|l|l|}
1 & 1 \\
\end{tabular} & 11 & & 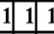 & & 1 & & & \\
\hline 2 Sensory augmentation & & & & & & & & & & & 1 & & & & & & & \\
\hline 2 Forward collision avoidance & & & & & & & & $1 \mid 1$ & 1 & 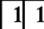 & $\begin{array}{lll}1 & 1 & \\
\end{array}$ & & 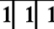 & & 1 & & & \\
\hline 2 Adaptive cruise control & & & & & & & & & & & & $1 / 1$ & & & & & & \\
\hline 2 Driver monitoring & & & & & & & & & 1 & \begin{tabular}{l|l}
1 & 1 \\
\end{tabular} & & \begin{tabular}{l|l}
1 & 1 \\
\end{tabular} & $1 \quad 1$ & & 1 & & & \\
\hline 2 Entertainment \& general information & & & & & & & & & & & & & 101 & & & & & \\
\hline 2 Notification sign information & & & & & & & & & 1 & & 1 & 1 & $\begin{array}{lll} & 1 \\
\end{array}$ & & & & & \\
\hline 3 Backing aid & & & & & & & & & & & & & & 1 & & & & \\
\hline 3 Automated toll collection & & & 1 & & & & & & & & & & & 1 & & & & \\
\hline 3 Heating, ventilation, air cond. \& noise & & & & & & & & & & & & & & 1 & 1 & & & \\
\hline 4 Railroad crossing collision avoidance & & & & & & & & & & & & & & & \begin{tabular}{l|l|}
1 & 1 \\
\end{tabular} & \begin{tabular}{l|lll}
1 & 1 \\
\end{tabular} & 11 & \\
\hline 4 Broadcast services/attractions & & & & & & & & & & & & & & & 1 & & & \\
\hline 4 Immediate hazard warning & & & & & & & & & & & & & & & 1 & \begin{tabular}{l|l}
1 & 1 \\
\end{tabular} & 11 & \\
\hline 4 Real-time communication & & & & & & & 1 & & & & & & & & & 1 & & \\
\hline 4 Asynchronous communication & & & & & & & & & & & & & & & & 1 & & \\
\hline 4 Interactive entertainment & & & & & & & & & & & & & & & & & 1 & \\
\hline 5 Vehicle condition monitoring & & & & & & & & & & & & 1 & & & 1 & & & \\
\hline 5 Manual aid request & & & & & & & & & & & & & & & & & & \\
\hline 5 Post-trip summary & & & & & & & & & & & & & & & & & 1 & 1 \\
\hline 5 Automatic aid request & & & & & & & & & & & & & & & & & & \\
\hline
\end{tabular}

Functional integration addresses important considerations ignored by the physical integration of a system. Functional integration transcends physical integration by combining functions in a manner that reduces demands on the driver. Network analysis of function centrality and function groups illustrates how this approach can guide integration of in-vehicle information systems to produce system designs that enhance, rather than degrade, driving safety and driver acceptance. Measures of centrality identify 
functions that play a particularly important role in overall system performance. Facilitating information flow between central functions will enhance system capabilities and reduce cognitive demands associated with reading, remembering and entering information on the part of the driver. For example, functions with high input centrality merit particular attention to minimise data entry. If information does not automatically flow to functions such as dynamic route selection, the drivers will have to finish the design and enter the data manually.

Cluster analysis identifies groups of functions that merit consideration as an aggregate, with common inputs/outputs. Clusters of functions may represent meta-functions whose operation is more meaningful and useful than individual functions. For example, the group of functions that includes trip planning, multi-mode travel coordination, pre-drive route and destination selection, road condition information, and contact search and history represents a group of functions that share information and would likely provide more value to the driver if considered together rather than separately. Specifically, integrating address information from the contact search and history function with the pre-drive route and destination selection function could eliminate the tedious address entry task that is commonly required with most route guidance systems. A specific example of how these functions might be integrated is a communicative navigation system. With such a system, a traveller might get into a rental car at the airport and the communicative navigation system would receive data from the traveller's PDA trip planning function regarding her hotel for that evening and automatically enter it as the destination. Similarly, when the traveller gets into the car the next morning the navigation system would receive data from the PDA's contact search and history function regarding the first business meeting of the day and automatically enter that as the destination. Destination entry requires substantial attention and so it can easily distract drivers if they try to enter destinations while they are driving (Eby and Kostyniuk, 1999). A communicative navigation system that integrates information from related functions could greatly enhance safety by reducing the need to enter destinations manually.

One particularly important finding is that the driver monitoring function plays a central role. Driver monitoring uses the state of other functions, the roadway environment and the driver's physiological state to estimate the driver's cognitive state and ability to accommodate additional information processing demands. The links between the driver monitoring function and the collision warning functions suggests that the timing of warnings should depend on the driver's cognitive state rather than simply the proximity of the collision threat. A specific example of how these functions might be integrated is an adaptive collision warning system. With such a system, the threshold for issuing a collision warning depends not only on the kinematics of the driving situation (relative distance, velocity and acceleration), but also on the degree to which the driver is attending to the roadway. A potential collision event might warrant a warning if the driver is inattentive, but not if the driver is attending to the road. An adaptive collision warning system that links the driver monitoring function with the collision warning functions to adjust the warning threshold according to the driver state could greatly reduce the number of unnecessary warnings and provide drivers with more time to react in situations that merit warnings. If an adaptive collision warning system was able to provide a slightly earlier warning to distracted drivers the safety benefit could be substantial (Lee et al., 2002). 
In summary, a matrix-based analysis to support functional integration of IVIS addresses three general problems that confront designers. First, it helps avoid forcing drivers to finish the design by requiring drivers to enter information that could be transferred automatically. Avoiding unnecessary data entry is a critical safety issue if the driver has to enter the information while driving. Secondly, it makes it easier to build a group of functions in which synergistic relationships can make the value of the group exceed the sum of the value of the individual functions. Pairing information regarding appointments and contacts with route guidance could be much more useful than the generic destination options. Thirdly, it helps provide a context to guide the behaviour of functions. Tuning warnings according to driver state may reduce driver annoyance associated with unhelpful warnings.

\subsection{Matrix analysis can guide graphical rendering of functions and information flows}

Beyond the design implications derived from quantitative analysis of the matrix of information flows, the analysis of groups and centrality can also guide drawing complex diagrams. Using matrix operations to identify clusters and central functions highlights functions that should be placed near each other in a diagram. Functions with high output centrality should be placed on the left of the diagram and high input centrality should be placed on the right. Placing functions of high total centrality in the centre of the diagram and physically grouping functions according to groups identified through matrix manipulation can greatly enhance the interpretation of complex systems. Such arrangements can also aid analysts in identifying information flows and interactions between functions that had not been previously considered and which might not have been readily apparent from the matrix manipulation.

\subsection{Considerations for a more precise description of function centrality and clusters}

This paper presented a simple binary characterisation of information flows to assess function centrality and clusters. The function analysis performed here did not consider the degree of criticality of information flows. Some information flows enhance convenience and others serve safety critical functions - it would be useful to acknowledge these distinctions in the measures of centrality. Nor did this analysis consider frequency of use: some information flows are frequently exercised while others are almost never exercised. Failure to consider the frequency of use as a measure of the relative importance of an information flow can distort estimates of criticality and centrality. For example, trip planning emerged as a highly central function even though it is likely to be used very infrequently. Trip planning supports multi-day travel. For most people this function would be used far less frequently (less than once per month) than pre-driver route or destination selection. Function analysis could be augmented to consider criticality and frequency of use to define magnitudes of information flows.

Complete specification of all the information flows for a complex system may not be practical for many design situations. In this situation, a subset of the functions could be analysed and useful insights could be gained. A comprehensive analysis of all possible functions is not required to identify important combinations of functions and important information flows. Likewise, this approach provides a robust mechanism for examining 
system properties. Overlooking important information flows will certainly distort the resulting measures of centrality and grouping of functions, but the analysis can highlight assumptions and make the missing information flows more obvious than they might otherwise be. An important contribution of this approach is that it helps designer arrange and analyse the functions in a systematic manner. This matrix-based analysis reveals missing information more readily than a more arbitrary arrangement and intuitive analysis. Recent development in the area of network analysis suggest that a growing array of analytic techniques will offer new ways to think about complex networks of functions (Blackhurst, Wu and O'Grady, 2004; Helbing and Kuhnert, 2003; Strogatz, 2001; Thelwall, 2003).

\section{Conclusions}

Examining IVIS functions using network analysis techniques demonstrates several important points relevant to the design of effective integrated information systems. First, the network analysis demonstrates the efficacy of matrix manipulation as a complement to the more common graphical representation of information flows. This is particularly important as the complexity of a system increases. For example, a system with as few as five functions may have as many as 25 information flows; graphical representation for systems having more than 20 functions can easily become unwieldy. In addition, network analysis can identify central functions and clusters of functions, which can then be used to guide the creation of graphics that are more likely to provide insight into system structure. Secondly, the functional integration supported by network analysis can avoid the problems that might plague a system design that considers only physical integration. A focus on physical integration is unlikely to address the interconnections required to support drivers in a safe and efficient manner. In fact, physical integration without functional integration may undermine driving safety. Thirdly, the analysis of function centrality and clusters can yield specific design concepts that combine functions in ways that are likely to enhance driver safety and satisfaction - the communicative navigation and adaptive collision warning systems, as examples. The analysis of IVIS functions shows the information flows that link functions, which designers must consider to create an integrated system. Information flows that are not included in the design may require the driver to 'finish the design', i.e., to manually support unintegrated information flows. This could result in an additional cognitive burden that might distract drivers with unnecessary data entry, annoy them with inappropriate warnings, or fail to provide the utility envisioned by the designers.

\section{Acknowledgements}

This research was funded by Federal Highway Administration contract DTFH 61-99-Y-30062 to Oak Ridge National Laboratory and by DTFH61-92-C-00102 to Battelle Human Factors Transportation Center. Thomas Granda was the Contracting Officer's Technical Representative for this project. 


\section{References}

Bavelas, A. (1948) 'A mathematical model for group structure', Human Organization, Vol. 7, pp.16-30.

Blackhurst, J., Wu, T. and O'Grady, P. (2004) 'Network-based approach to modelling uncertainty in a supply chain', International Journal of Production Research, Vol. 42, pp.1639-1658.

Borgatti, S.P., Everett, M.G. and Freeman, L.C. (1992) 'UCINET IV Version 1.00', Analytic Technologies, Columbia.

Cook, R.I., Woods, D.D. and Howie, M.B. (1990) 'The natural history of introducing new information technology into a high-risk environment', presented at Proceedings of the Human Factors Society 34th Annual Meeting, Orlando, FL.

Eby, D.W. and Kostyniuk, L.P. (1999) 'An on-the-road comparison of in-vehicle navigation assistance systems', Human Factors, Vol. 41, pp.295-311.

Freeman, L.C. (1978) 'Centrality in social networks conceptual clarification', Social Networks, Vol. 1, pp.215-239.

Glover, F. (1977) 'Heuristics for integer programming using surrogate constraints', Decision Sciences, Vol. 8, pp.156-166.

Helbing, D. and Kuhnert, C. (2003) 'Assessing interaction networks with applications to catastrophe dynamics and disaster management', Physica A: Statistical Mechanics and Its Applications, Vol. 328, pp.584-606.

Jameel, A., Stuempfle, M., Jiang, D. and Fuchs, A. (1998) 'Web on wheels: toward internet-enabled cars', Computer, Vol. 31, No. 69.

Kirwan, A. (Ed.) (1992) A Guide to Task Analysis, Taylor \& Francis, Washington, DC.

Kusiak, A. (1999) Engineering Design: Products, Processes, and Systems, Academic Press, New York.

Lee, J.D. (1997) 'A functional description of ATIS/CVO systems to accommodate driver needs and limits', in Noy, Y.I. (Ed.): Ergonomics and Safety of Intelligent Driver Interfaces, Lawrence Erlbaum Associates, Mahwah, NJ, pp.63-84.

Lee, J.D., McGehee, D.V., Brown, T.L. and Reyes, M.L. (2002) 'Collision warning timing, driver distraction, and driver response to imminent rear-end collisions in a high-fidelity driving simulator', Human Factors, Vol. 44, pp.314-334.

Lee, J.D. and Sanquist, T.F. (2000) 'Augmenting the operator function model with cognitive operations: assessing the cognitive demands of technological innovation in ship navigation', IEEE Transactions on Systems, Man, and Cybernetics- Part A: Systems and Humans, Vol. 30, pp.273-285.

Linstone, H. and Turoff, M. (1975) The Delphi Method: Techniques and Applications, Addison-Wesley, New York.

Luce, R.D. and Perry, A.D. (1949) 'A method of matrix analysis of group structure', Psychometrika, Vol. 14, pp.95-116.

Nieminen, J. (1974) 'On centrality in a graph', Scandinavian Journal of Psychology, Vol. 15, pp.322-336.

Norman, D.A. (2003) 'Interaction design for automobile interiors', JND, http://www.jnd.org/dn.mss / interaction_design_f.html

Rasmussen, J. (1985) 'The role of hierarchical knowledge representation in decisionmaking and system management', IEEE Transactions on Systems, Man, and Cybernetics, SMC/15, pp.234-243.

Rasmussen, J. (1986) Information Processing and Human-Machine Interaction: an Approach to Cognitive Engineering, North Holland, New York.

Rouse, W.B. and Cody, W.J. (1988) 'On the design of man-machine systems: principles, practices and prospects', Automatica, Vol. 24, pp.227-238. 
Sarter, N.B. and Woods, D.D. (1995) 'How in the world did we ever get in that mode? Mode error and awareness in supervisory control', Human Factors, Vol. 37, pp.5-19.

Strogatz, S.H. (2001) 'Exploring complex networks', Nature, Vol. 410, pp.268-276.

Thelwall, M. (2003) 'Web use and peer interconnectivity metrics for academic websites', Journal of Information Science, Vol. 29, pp.1-10.

Turban, E. and Tan, M. (1993) 'Methods for knowledge acquisition from multiple experts: an assessment', International Journal of Applied Expert Systems, Vol. 1, pp.101-119.

Vicente, K.J. (1999) Cognitive Work Analysis: Towards Safe, Productive, and Healthy Computer-Based Work, Lawrence Erlbaum Associates, Mahwah, NJ.

Wasserman, S. and Faust, K. (1994) Social Network Analysis: Methods and Applications, Cambridge University Press, New York.

Woods, D.D. (1994) 'Automation: apparent simplicity, real complexity', in Mouloua, M. and Parasuraman, R. (Eds.): Human Performance in Automated Systems: Current Research and Trends, Lawrence Erlbaum Associates, Hillsdale, NJ , pp.1-7. 